\title{
Symbols are not uniquely human
}

SidARTA RibeIRo*1 ${ }^{*}$ ANGElo LOUla ${ }^{* 2,3}$, IVAN DE ARAÚJO ${ }^{4}$, RiCARdo GUdWIN ${ }^{2}$ AND JoÃo QUEIROZ $^{2,5}$

1 International Institute of Neuroscience of Natal (IINN), Rua Professor Francisco Luciano de Oliveira, 2460, Bairro Candelária, 59066-060, Natal, RN, Brazil

2 Dept. Computer Engineering and Industrial Automation, School of Electrical and Computer Engeneering (FEEC), State University of Campinas (UNICAMP)

Av. Albert Einstein, 400

13083-852, Campinas, SP, Brazil

3 Informatics Area, Department of Exact Sciences (DEXA), State University of Feira de Santana (UEFS)

Km 3, BR116, Campus Universitário

44031-460, Feira de Santana, BA, Brazil

4 Dept. Neurobiology, Duke University Medical School

Box 3209

27710, Durham, NC, USA

5 Institute of Biology, Federal University of Bahia (UFBA), Salvador, BA, Brazil

Rua Barão de Geremoabo, 147 Campus de Ondina

40170-290, Salvado, BA, Brazil

* These authors contributed equally

Corresponding Author: João Queiroz

queirozj [at] dca.fee.unicamp.br

Phones +55 (19) 3788-3819, +55(19) 3788-3706

Fax +55 (19) 3289-1395 


\section{Symbols are not uniquely human}

Abstract. Modern semiotics is a branch of logics that formally defines symbol-based communication. In recent years, the semiotic classification of signs has been invoked to support the notion that symbols are uniquely human. Here we show that alarm-calls such as those used by African vervet monkeys (Cercopithecus aethiops), logically satisfy the semiotic definition of symbol. We also show that the acquisition of vocal symbols in vervet monkeys can be successfully simulated by a computer program based on minimal semiotic and neurobiological constraints. The simulations indicate that learning depends on the tutor-predator ratio, and that apprenticegenerated auditory mistakes in vocal symbol interpretation have little effect on the learning rates of apprentices (up to $80 \%$ of mistakes are tolerated). In contrast, just $10 \%$ of apprentice-generated visual mistakes in predator identification will prevent any vocal symbol to be correctly associated with a predator call in a stable manner. Tutor unreliability was also deleterious to vocal symbol learning: a mere 5\% of "lying" tutors were able to completely disrupt symbol learning, invariably leading to the acquisition of incorrect associations by apprentices. Our investigation corroborates the existence of vocal symbols in a non-human species, and indicates that symbolic competence emerges spontaneously from classical associative learning mechanisms when the conditioned stimuli are self-generated, arbitrary and socially efficacious. We propose that more exclusive properties of human language, such as syntax, may derive from the evolution of higher-order domains for neural association, more removed from both the sensory input and the motor output, able to support the gradual complexification of grammatical categories into syntax.

Keywords: symbols, semiotic and neurobiological constraints, computer simulation of symbol learning

\section{Introduction}

The semiotics of Charles S. Peirce has long been suspected a powerful tool for the investigation of language and communication (Ransdell 1977; Noble, Davidson 1996; Deacon 1997, 2003). In this system, all communication occurs by way of three inter-dependent but separate elements: object, sign and effect on an interpreter (interpretant) (Peirce 1998). In Peircean semiotics, meaning occurs when a sign communicates an object to an interpreter. Any description of meaning involves an irreducible (non decomposable) relation constituted by three elements: a sign, an object, which the sign represents, and an intepretant, which is the outcome of the sign on the interpreter. A sign can only be of three kinds - icons, indexes, and symbols - according to the relationship established with its object. Icons are signs which stand for their objects through similarity or resemblance. In contrast, indexes establish a spatiotemporal physical correlation to their objects. Finally, in a symbolic relation, the sign stands for its object through a determinative relation of law, rule or convention. According to Peirce $(1958, \S 2.307)$, a symbol is "a Sign (q.v.) which is constituted a sign merely or mainly by the fact that it is used and understood as such, whether the habit is natural or conventional, and without regard to the motives which originally governed its selection.” In this symbolic sign 
process, the object which is communicated to the interpretant through the sign is a lawful relationship between a given kind of sign and a given type of object. Generally speaking, a symbolic sign communicates a law to the interpretant as a result of a regularity in the relationship between sign and object.

Despite its internal consistency, semiotics resonated little within the neuroscience community devoted to the understanding of language and communication. In recent years, an attempt to bridge semiotics and neurobiology concluded that symbols do not naturally occur in species other than humans, configuring the 'symbolic species' theory (Deacon 1997, 2003). The idea that the Homo Sapiens is the only extant species that employs symbols frontally collides with several lines of evidence regarding animal behavior and evolution (Struhsaker, Hunkeler 1971; Savage-Rumbaugh, Rumbaugh et al. 1978; Seyfarth, Cheney et al. 1980; Lieberman 1984; Richards, Wolz et al. 1984; Savage-Rumbaugh, Romsky et al. 1989; Griffin 1992; Langs, Badalamenti et al. 1996; Hauser 1997; Lieberman 1998; Whiten, Goodall et al. 1999; Hauser 2000; Xia, Emmerton et al. 2001; Pepperberg 2002; Bergman, Beehner et al. 2003; Kaminski, Call et al. 2004). Furthermore, we have previously argued that this theory is in fact incongruent with Peircean mature semiotics, i.e. the late philosophical production of $\mathrm{C}$. S. Peirce (Queiroz, Ribeiro 2002). In fact, the 'symbolic species' theory borrows from his semiotics little more than definitions of the three basic types of signs (icons, indexes and symbols), falling short of proposing what their neural correlates may be. As a consequence, the theory fails to explore the vast semiotic framework to solve questions regarding the emergence of symbolic language.

To re-assess the use of symbols in non-human animals, we subjected the well-known case of vocal communication displayed by African vervet monkeys (Cercopithecus aethiops) to a semiotic analysis in terms of sign classification. These primates possess a sophisticated repertoire of vocalizations used for intra-specific alarm purposes regarding imminent predation on the group. Field studies have revealed three main kinds of alarm-calls separately used to warn about the presence of (a) terrestrial stalking predators such as leopards, (b) aerial raptors such as eagles, and (c) ground predators such as snakes (Struhsaker 1967; Seyfarth, Cheney et al. 1980). Adult vervets produce these calls only in reference to the presence of predators. Such calls, when heard by other adults, motivate whole-group escape reactions that are specific to predator type. For instance, when a "terrestrial predator" call is uttered, vervets escape to the top of nearby trees; "aerial predator" calls cause vervets to hide under trees, and "ground predator" calls elicit rearing on the hindpaws and careful scrutiny of the surrounding terrain. 
While adults share a code for predator reference, infant vervet monkeys babble alarm calls in response to a variety of animals (predators and non-predators), as well as to inanimate objects such as falling leaves etc. As a consequence, adults pay little attention to infant calls (Cheney, R. 1990; Seyfarth, Cheney 1997). The progressive specificity of alarm-call production as vervets grow older indicates that a great deal of learning is necessary before these calls can be used in the proper context (Seyfarth, Cheney 1980, 1986). Indeed, field experiments in which predator-specific alarm-calls were played from loudspeakers to groups of wild vervets monkeys showed that adult individuals first responded to playbacks of alarmcalls by looking around in search of a referent (predator). Remarkably, even though this referent was always absent, adult animals consistently fled away to nearby refuges according to the specific type of alarm-call played. Infant monkeys, on the other hand, responded poorly to playbacks, and teenagers displayed intermediate behaviors (Seyfarth, Cheney 1980, 1986; Cheney, R. 1990; Seyfarth, Cheney 1997). The assumption that the mapping between calls and predators can be learned is also supported by the observation that cross-fostered macaques, although unable to modify their call production, "did learn to recognize and respond to their adoptive mothers' calls, and vice versa" (Cheney, Seyfarth 1998).

Taken together, these experiments indicate that the meaning of vervet monkey alarmcalls can be learned even in a highly noisy environment through social interactions with multiple tutors.

\section{Methods}

To gain insight into the mechanisms of vocal communication in vervet monkeys, we first modeled a minimum brain designed to satisfy very basic neurobiological constraints, common in principle to any animal with a nervous system. This model brain is composed of four interconnected representation domains (RD), in relationship with a perceptible external world containing images of predators, alarm-calls, and images of other behaving monkeys (Fig. 1a). Separate domains of primary sensory representation (RD1S) comprise two different sensory modalities, visual and auditory, each of them composed of multiple relays connected in series by reciprocal connections. RD1S domains are the input layers of the system, and are connected to an integrative domain of secondary sensory association (RD2), defined as the only domain capable of evaluating the behavioral value of a given association, so as to increase or decrease its strength based on past outcome. Once evaluated by RD2, behavioral value can affect motor representation domains able to generate the behavioral output (RD1M). In a mammal, RD1S and RD1M domains respectively comprise primary sensory and motor 
areas in the mesencephalon, diencephalon and telencephalon; the RD2 domain includes associative areas of the cerebral cortex, the hippocampus and the amygdala.

a

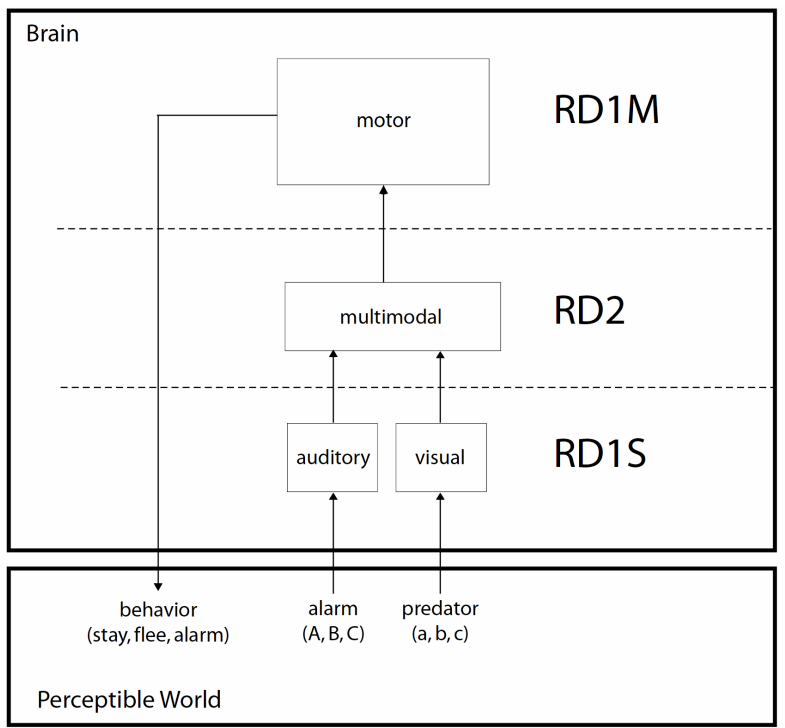

C

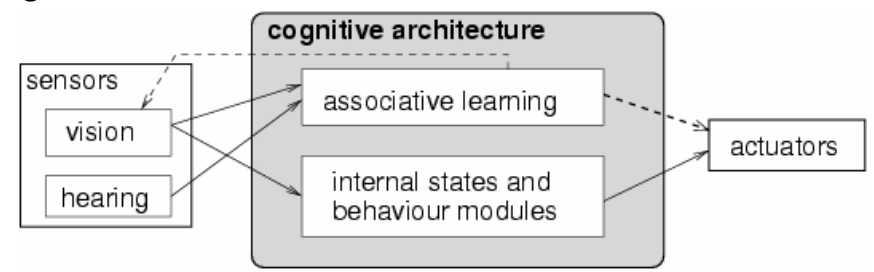

$\boldsymbol{d}$

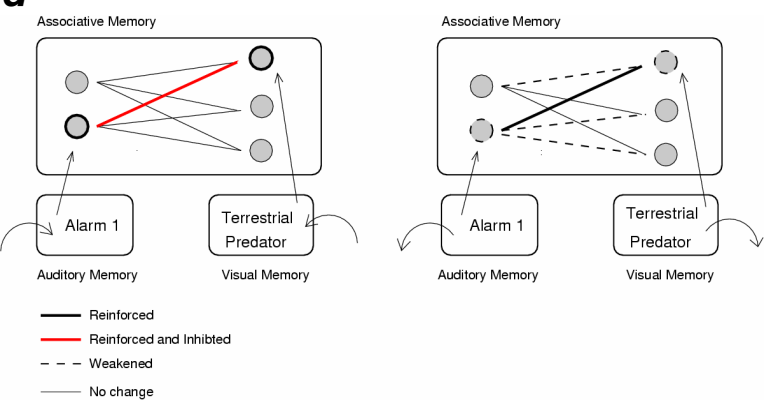

b

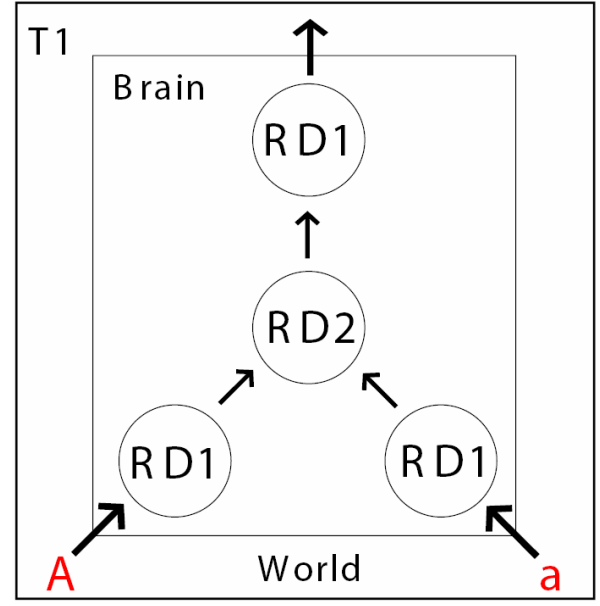

e

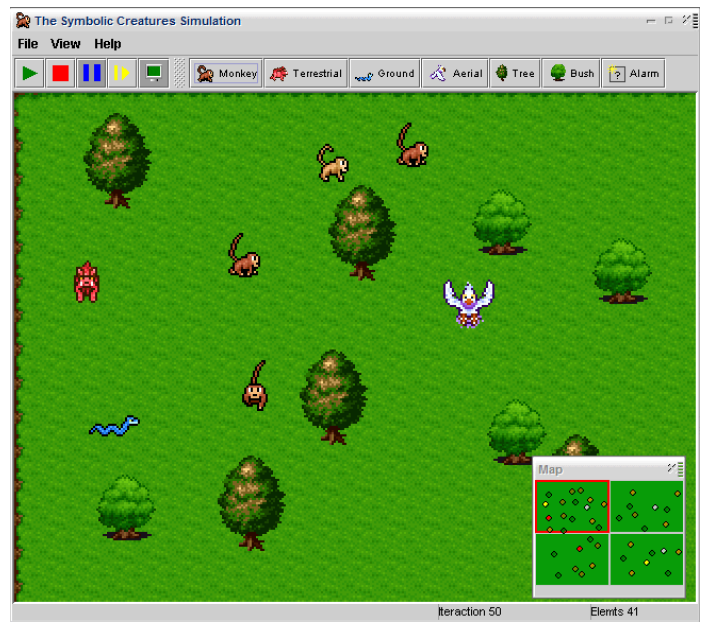

Figure 1: Concepts underlying a neurosemiotic simulation. a) Minimum brain architecture chosen for our simulations, containing two domains for primary sensory representation (RD1S), one domain for secondary sensory association (RD2) and one domain for primary motor representation (RD1M). b) Simplified representation of the minimum brain architecture, in which circles stand for domains of representation in the monkey brain (RDs). c) Control architecture of the apprentice creature used in our simulations. Each contains multiple relays in RD1S and RD1M $d$ ) Each apprentice contains also some relays dedicated to the association between images and sounds, i.e. RD2.e) Picture of the simulation console showing preys, predators, bushes and trees.

In this model, sign, object and interpretant are well-defined physical entities of brain or world, in a given instant t. Objects and interpretants occur as elements of the observable world (calls, images) or as neural representations in a brain domain, i.e. ensembles of co- 
activated neurons. Signs, on the other hand, correspond to the patterns of anatomical mapping and functional connectivity that link neural representations in different brain domains to the external world or among themselves. Thus, the activation of a specific neuronal ensemble A in a brain domain $A^{\prime}$ (object) is conveyed by way of a given connectivity pattern (sign) so as to activate a corresponding neuronal ensemble B within another brain domain B' (interpretant). Information flows uni-directionally in our simplified model: objects of the external world are conveyed to the brain by way of signs, which can be classified as icons, indexes, symbols (as we shall see later on). These are interpreted as neural representations in RD1S configuring another object that is then presented via another sign to RD2, and so on to RD1M until a behavior is produced in the world, thus becoming an object available to any observer (Fig. 1b).

To further explore the underlying mechanisms for the acquisition of alarm calls, we implemented an agent-based simulation of a prey-predator community inspired by the vervetmonkey case, as previously described elsewhere (Loula, Gudwin et al. 2003; Loula, Gudwin et al. 2004) (also see www.dca.fee.unicamp.br/projects/artcog/symbcreatures/). In our simulation, each creature acting in the virtual environment has an independent control system, being classified as either prey or predator. Preys are further characterized as tutors or apprentices, depending on whether they know the repertoire that assigns one alarm-call type to each predator class. These autonomous artificial creatures were equipped with sensors (visual and auditory) and actuators (e.g. move, vocalize, change gaze direction), controlled by multiple parallel behavior modules such as wandering, visual scanning, fleeing or chasing. Working and associative memories were implemented in apprentice creatures, and each memory instantiation was classified as pertaining to one of the four representational domains defined above. The control architectures of the artificial creatures were in essence the same as the minimum brain (Fig. 1a). Although multiple serial relays were necessary to implement all the visual, auditory, motor and decisional functions attributed to the artificial creatures (Fig. 1c), cross-modality associations were only implemented by way of associative memory domains able to perform RD2 functions (Fig. 1d). In this model, memory formation in RD2 follows Hebbian associative learning principles (Hebb 1949), one of the simplest learning mechanisms, which can be widely found in non-human species. During simulation runs, tutor preys vocalize three specific alarms in the presence of predators while apprentices try to establish the relation between different alarms heard and environment objects and creatures seen.

When an apprentice prey receives a visual or auditory stimulus, stimulus-related information is kept in the respective working memory for a few instants, allowing different 
stimuli received in close instants to co-occur in memory. The associative memory uses the cooccurrence of stimuli in both visual and auditory memories to strengthen associations (with values between 0 and 1). As soon as auditory and visual stimuli are jointly presented, their association is strengthened and further changes are forbidden until both stimuli leave the working memory, avoiding thus multiple reinforcements. Any stimulus in the visual memory can be associated with any stimulus in the auditory memory, and therefore apprentices may actually learn spurious associations, such as that between an alarm call and the image of another prey, or a tree. Weakening adjustments are conducted when it is detected that a stimulus does not co-occur with another one. When a stimulus (of either modality) is received but no other stimuli (of the other modality) are perceived in close temporal proximity, the associations between the received stimulus and all non-perceived stimuli are weakened as soon as the received stimulus leaves the working memory. To allow the stabilization of stronger associations, we also implemented a lateral inhibition mechanism, which changes the strengthening and weakening rates based on the value of the strongest associations. When any particular association is changed, its adjustment rate is altered according to the strongest association among all associations with the same stimulus. As a consequence, high strength of the strongest association determines low strengthening/high weakening rates for all the competing associations ${ }^{1}$.

To test the robustness of symbolic learning by artificial preys, we varied three parameters affecting sign interpretation: visual noise, auditory noise and tutor reliability. Visual noise was simulated as the probability of an apprentice interpreting any given image as a predator. This probability was assessed every time an image was perceived by each apprentice, and the occurrence of visual noise resulted in a 1/3 chance of misidentifying the visual stimulus as belonging to one of the 3 classes of predators. Auditory noise was modeled as a probability that an apprentice would interpret an alarm-call properly vocalized by a tutor

\footnotetext{
${ }^{1}$ The actual adjustment formulas are:

- strengthening, given a visual stimulus $i$ and a hearing stimulus $j$ in the work memories
} $s_{i j}(k+1)=s_{i j}(k)+0.1\left(1.0-\left(m s_{j}(k)-s_{i j}(k)\right)\right)+0.0$, where $\operatorname{ms}_{j}(k)=\max _{i} s_{i j}(k)$

- weakening, for a visual stimuli $i$ leaving the working memory $\forall j$ associated with $i$ with adjustments not prohibited, $s_{i j}(k+1)=s_{i j}(k)-0.1\left(m s_{j}(k)-s_{i j}(k)\right)-0.01$

- weakening, for a dropped hearing stimuli $j$ leaving the working memory $\forall i$ associated with $j$ with adjustments not prohibited, $s_{i j}(k+1)=s_{i j}(k)-0.1\left(m s_{j}(k)-s_{i j}(k)\right)-0.01$

The association strength value between $i$ and $j$ is represented as $s_{i j}$. The 0.01 value is used to allow a minimum change in associations. 
as a different kind of alarm. This probability was tested each time an apprentice heard an alarm-call, and upon verification it determined a misinterpretation as a $4^{\text {th }}$ or $5^{\text {th }}$ type of alarmcall (50\% chance each). Finally, tutor reliability was simulated as a probability that tutors could vocalize any of the three types of alarm-calls in the absence of predators; reliability was assessed at every iteration.

\section{Results}

We used our minimum model to implement a graphic simulation of vervet monkey intra-specific communication. Two extreme social categories were distinguished: infants were assumed to have no previous knowledge of the meaning of either alarm-calls or predator views. With no "a priori" memory in RD2 regarding these objects, infants initially react to any world scene with a default "stay" behavior. Adults, on the other hand, were considered to

fully understand the meaning of predator images and of the vocal communication repertoire shared by other adult members of the group. This means that adults have memories in RD2 that associate the neural representations of predators and alarm-calls to "escape" reactions and further alarm calling. The consequences of a single synchronous presentation of both alarm call and predator view to an infant and an adult are shown in Figure 2. Infants are initially unresponsive to both alarm calls and predator views (S for "stay"), but exposure to the escape reaction of adult tutors leads to learning (Fig. 2a) As expected, adults escape much earlier than infants in this situation (Fig. 2b). In both cases, alarm calls (signs) are either interpreted as icons (eg. the topographic activation of visual and auditory RD1S by predator image or alarm-call, respectively) or as an index (the non-topographic, spatio-temporal conjunction of information from both modalities in RD2 and therefore in RD1M). 
a
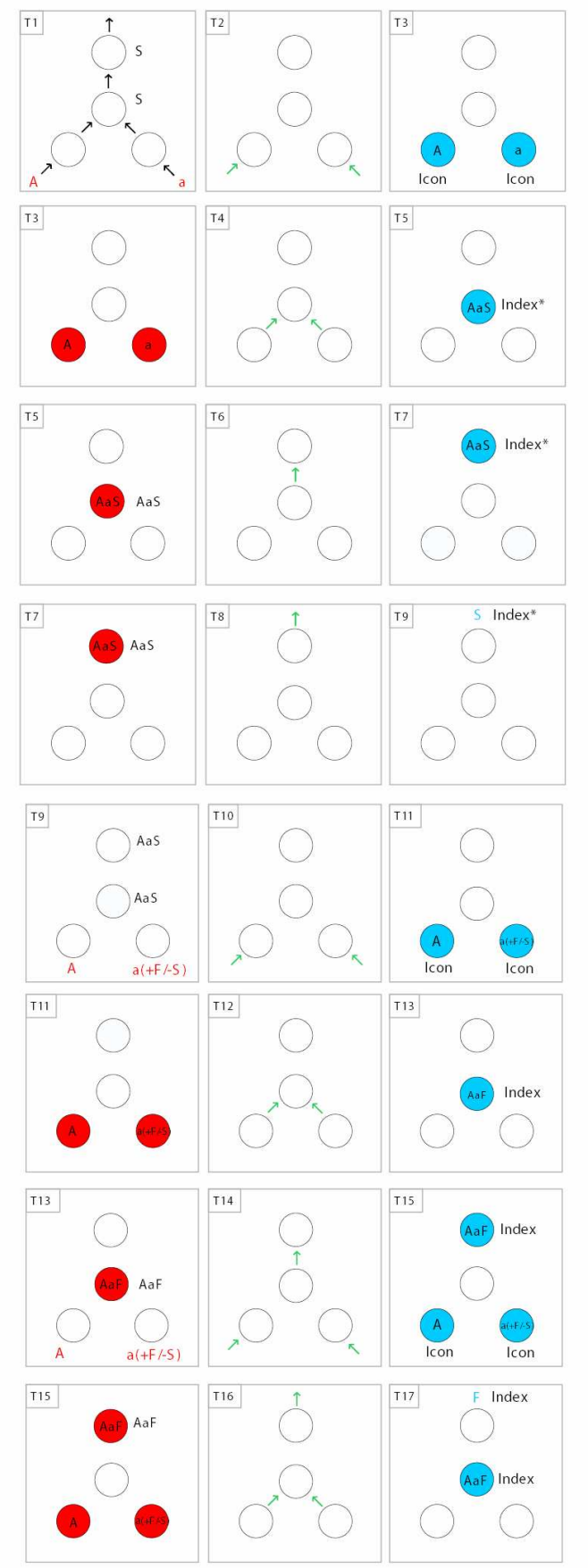

b

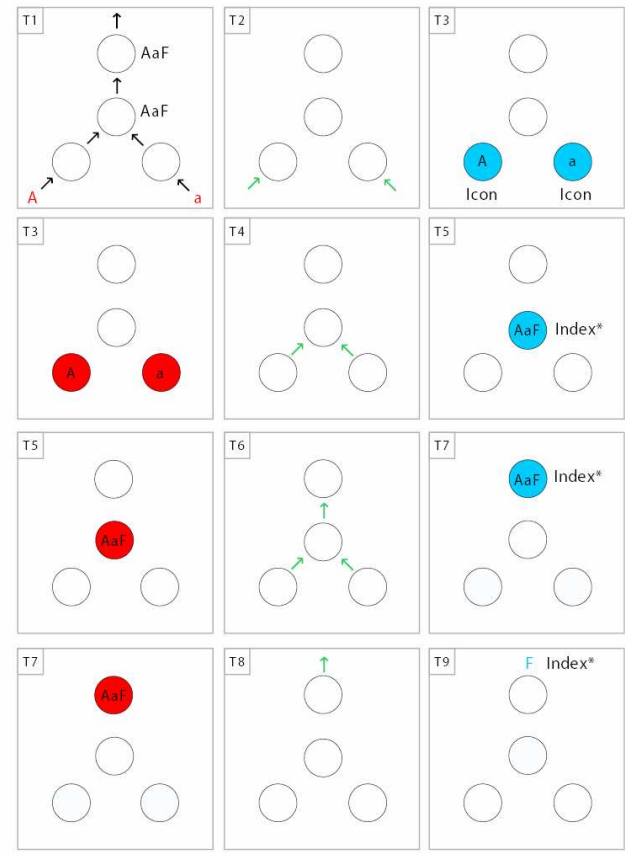

C

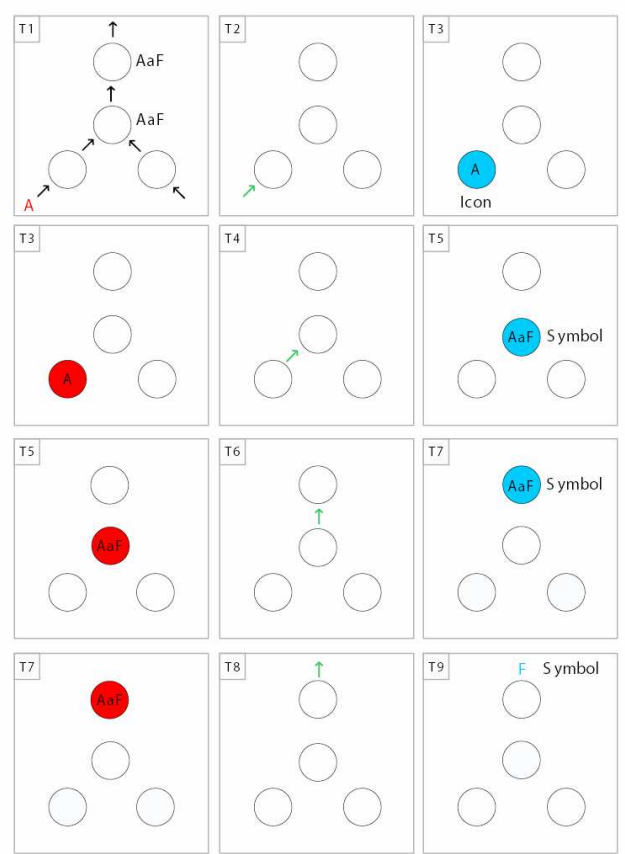

Figure 2: Storyboard of sign process of alarm call communication employing vervet monkeys' minimum brain architecture. Each frame is constituted of letters, arrows and circles. T1, T2, T3 etc represent instants in time. External objects presented to a monkey brain comprise predator images (a, b, c), corresponding alarm-calls vocalized by other monkeys (A, B, C), and reactive behaviors from neighboring monkeys that may be visible to other brains (F refers to "flee"; S refers to "stay"). Circles stand for domains of representation in the minimum brain (RDs). Circle colors indicate different types of neural representations according to their semiotic relationships - Red for object and Blue for interpretant. The white color designates a de-activated RD (circle) or the absence of an external object. Arrows represent signs, i.e. patterns of connectivity between brain areas, or between a brain area and the external world. Green arrows indicate instantiation of a connectivity pattern, i.e. the action of a sign translating from an object to an interpretant. Black arrows in T1 indicate latent (inactive) signs. Memory for a representation is indicated as letters outside the boundaries of circles in T1. Information about the particular identity of an external object is represented by outside letters in T1, T9 and T13 (arbitrary moments of 
occurrence); this information is preserved within the brain as indicated by letter inside circles thereafter. At T3, interpretants within RD1S become internal (neural) objects to be represented downstream, determining the repetition of T3 in the next frame, and so on. Every instantiation of a representation in RD2 causes a slight increase in memory of that representation. Every instantiation of a representation must be interpreted as either an Icon, or an Index, or a Symbol; Memory of a representation (A) of the object (a) is defined as the probability of observing (A) given a certain context of object presentation that might or not include (a). In addition, external objects may also carry the capacity to signify reward or punishment. This capacity (object value) is defined as positive and negative variables that can increase or decrease the memory of associated representations. -S refers to negative value imposed on brain representations associated with "stay"; +F refers to positive value imposed on brain representations associated with "flee". a) Infant simultaneously presented with predator image and alarm call. $b$ ), Adult simultaneously presented with predator image and alarm call. Once again an escape response is generated earlier (T9) than in infants (T17). This crucial symbolic step occurs in T5, when RD2 interprets the ascending iconic representation " $\mathrm{A}$ " as "AaF"). c) Adult presented with an alarm-call only.

Symbols emerged in our model when adults were presented with an alarm-call in the absence of a corresponding predator view, as in the playback experiment carried on by Seyfarth and Cheney (Seyfarth, Cheney et al. 1980) (Figure 2c). In this case, the lack of temporal pairing between alarm-call and predator image precludes interpretation of the alarm call as an index. According to the Peircean classification of signs, if the alarm-call operates in a specific way even in the absence of the external particular referent, then it must be interpreted as a symbol of a predator class and of a particular type of escape strategy (Queiroz, Ribeiro 2002). The transition from a sensory scan behavior after the alarm auditory perception to an escape reaction motivated solely by the alarm-call corresponds to the transition from an indexical (interpretation by spatio-temporal coincidence) to a symbolic process (interpretation mediated by law). The object of the sign, in the latter case, is not an object but a class of objects, and therefore does not need to exist as a singular event. To say that an alarm-call is a symbol of a type of predator is equivalent to say that this call evokes a brain representation (of any sensory modality or combination of modalities) that stands for the class of predators represented in a lawful and specific way. This symbolic relationship implies the memorized association of at least two lower-order representations (i.e. indices or icons) in a higher-order representation domain (RD2). The neurosemiotic diagrams shown in Fig. 2 constitute an evidence that vervet monkeys, as much as described in the current literature (Struhsaker 1967; Seyfarth, Cheney et al. 1980; Seyfarth, Cheney 1986; Cheney, R. 1990; Seyfarth, Cheney 1997), employ symbols in their intra-specific communication. 
a

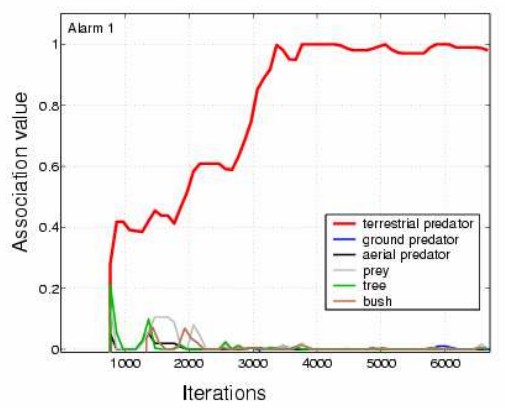

b

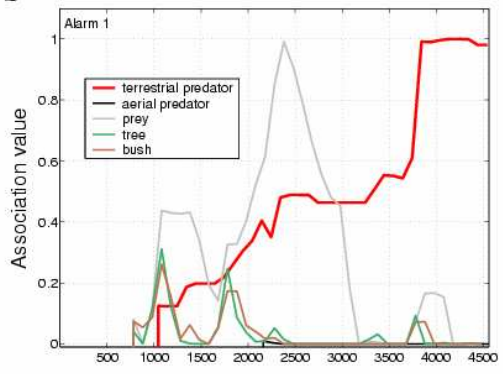

Tutor/Apprentice Ratio $=5$
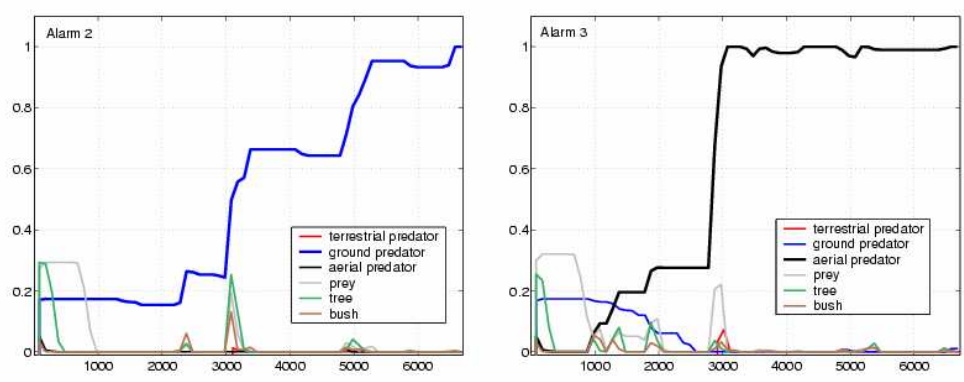

Tutor/Apprentice Ratio $=10$
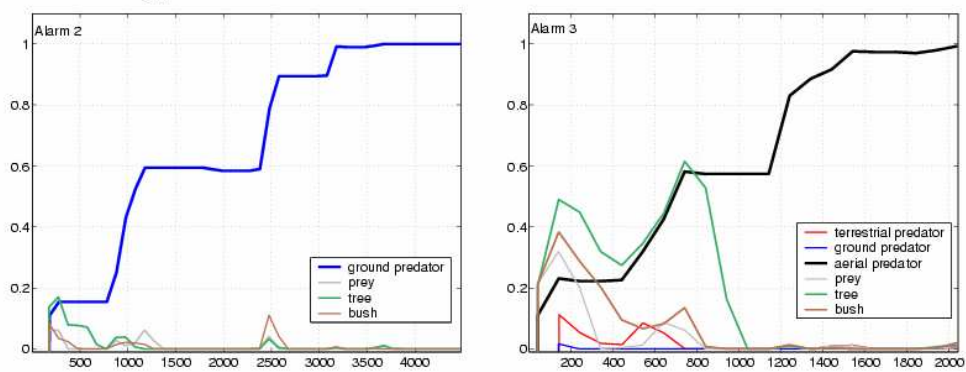

Figure 3: Naïve preys develop vocal symbol learning spontaneously after a few thousand iterations. The number of iterations required for learning has an inverse relationship with the tutor-apprentice ratio.
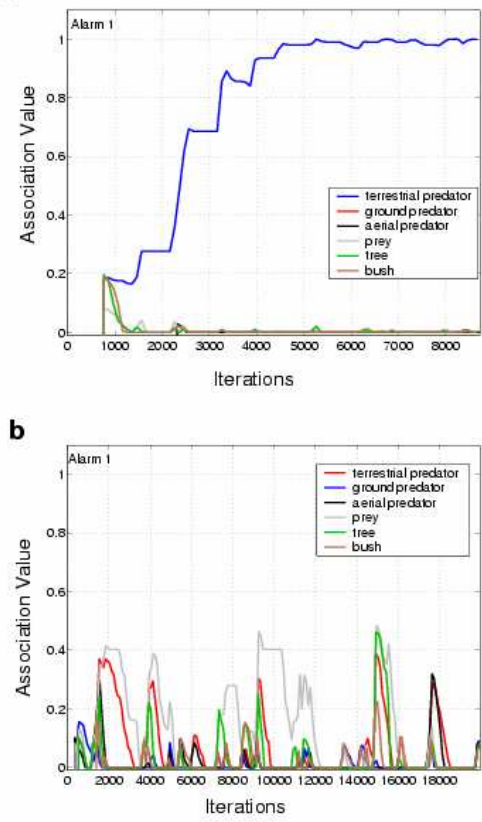

C

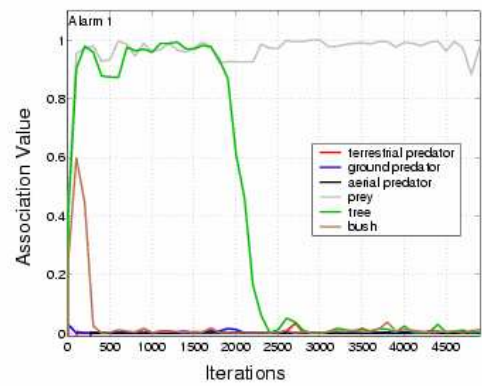

Auditory Noise $80 \%$

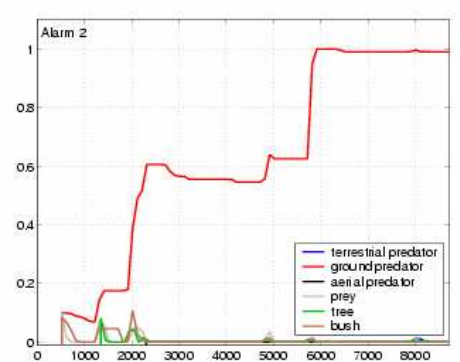

Visual Noise $10 \%$

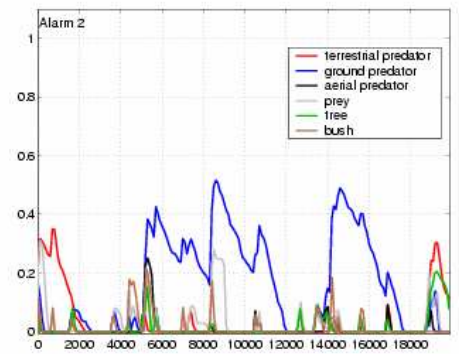

Tutor Reliability 5\%

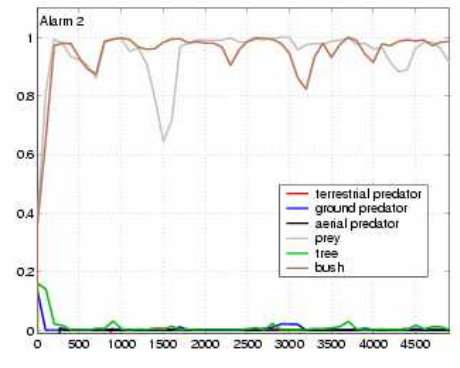

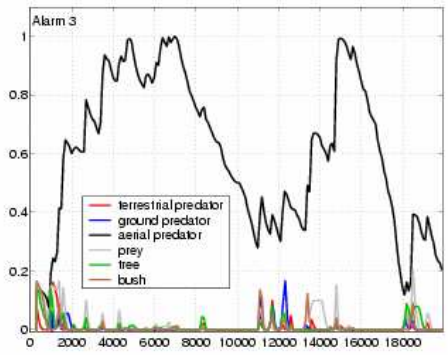
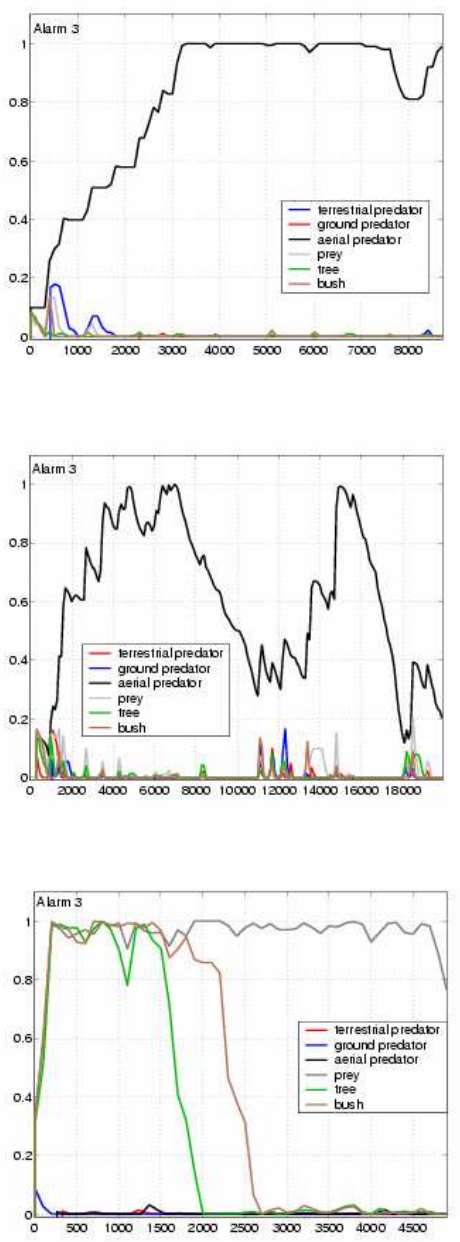

Figure 4: Vocal symbol learning is highly resistant to apprentice-generated auditory noise (a), but is strongly impaired by apprentice-generated visual mis-identification of predators (b) and by tutor unreliability (c). 
Next, we implemented a simulation of a prey-predator community to determine whether the neurosemiotic constraints described above could account for general symbol learning processes under more realistic conditions. Results show that apprentices were able to learn the symbolic association between alarm-calls and predator type over a finite number of iterations (figure 3a). As the number of tutors per apprentice decreased the learning process became slower, likely due to a decreased ratio of effective communication events over predatory events (figure $3 \mathrm{~b}$ ). It took from 1500 to 3750 iterations for artificial preys to learn in the first case and from 3000 to 5250 in the second case.

The introduction of noise in the system led to different outcomes depending on the noise source. Symbolic learning was highly resistant to auditory noise, which even at $80 \%$ rates was incapable of preventing the eventual learning of correct associations (Fig. 4a). In contrast, a mere $10 \%$ of visual noise proved to be destructive to symbolic learning, leading to unstable associations that shifted back and forth without reaching equilibrium (Fig. 4b). Also disruptive to learning was tutor unreliability, which led to the stabilization of incorrect associations even at 5\% levels (Fig. 4c).

\section{Discussion}

Our results demonstrate that symbols can evolve in a population of sensory-motor organisms equipped with classical associative learning, provided that the conditioned stimuli are self-generated, arbitrary and socially efficacious. This suggests that the biological prerequisites for the emergence of symbolic communication must extend well beyond the realm of human behavior, as observed in apes (Savage-Rumbaugh, Rumbaugh et al. 1978; SavageRumbaugh, Romsky et al. 1989; Langs, Badalamenti et al. 1996; Whiten, Goodall et al. 1999), monkeys (Struhsaker, Hunkeler 1971; Seyfarth, Cheney et al. 1980; Bergman, Beehner et al. 2003), dolphins (Richards, Wolz et al. 1984; Herman, Kuczaj et al. 1993), dogs (Kaminski, Call et al. 2004), and birds (Xia, Emmerton et al. 2001; Pepperberg 2002). In the coming years, the comprehensive investigation of symbolic competence in non-human animals should further falsify the notion that symbols are uniquely human.

Based on the field evidence presently available, grammar and syntax do not occur in vervet-monkeys, which seem to only have a small repertoire of signs not amenable to further composition. According to Peirce's mature semiotics, symbols can be further analysed in 3 subclasses, of which only one, termed argument, possesses the property of linguistic composition. Peirce developed a typology to describe "rudimentary" forms of symbols. In the absence of human experimenters and playback tricks, alarm calls vocalized by vervet 
monkeys represent classes of objects that exist in the real world. Therefore, these alarm calls are symbols interpreted as indices of the presence of the predator: In semiotic terminology, alarm-calls are dicent symbols, for the object of a dicent symbol is a 'general' interpreted as an 'existent' (Peirce 1998).

Our results are in line with the notion that symbolism preceded syntax in evolution (Bickerton 2003). The origin of grammaticality in the Homo lineage may fall within the early Pleistocene threshold for syntax emergence (around 1.8 million years ago) (Bickerton 2003), an evolutionary turning point towards the complexification of the hominid vocal apparatus that likely enabled an increase in symbolic repertoire, the gradual emergence of composite phonemes and words, and probably syntax by the Middle Paleolithic (Lieberman, Crelin 1971; Arensburg, Tillier et al. 1989; Arensburg, Tillier 1991). This process may have involved inter-specific interactions not yet considered. For instance, it is tempting to imagine the tutoring influence that highly vocal non-ape primates may have had on early hominids, as the latter transited from gestural to vocal language in the dangerous savannahs of the early Pleistocene (Vrba 1988a, b).

The results also indicate that vocal symbol learning in a prey-predator community is a robust phenomenon that develops over a wide range of tutor-predator ratios. In our simulations, even though the amount of iterations before symbol learning was in the order of 3000 , only a few dozen meaningful communicative interactions were crucial for learning to occur. It will be interesting to compare this observation with the actual number of iterations experienced by apprentice vervet-monkeys before learning is established. We also found that vocal symbolic learning in artificial creatures was highly resistant to auditory noise capable of impairing the correct identification of alarm-calls by apprentices. Auditory noise is very high in the real world, so it is encouraging that the artificial creatures were able to learn their language under more realistic acoustic conditions. On the other hand, learning became unstable when the visual identification of the predators was impaired. This suggests a strong selection pressure against visual deficits.

Perhaps the most interesting finding of our simulations was the striking disruption of learning provoked by relatively small amounts of tutor unreliability. This suggests a strong selection pressure against tutor unreliability, a prediction to be tested by field studies. Incidentally, direct evidence of symbolic communication in vervet monkeys, without the aid of playback tricks, would only be possible in case tutors lied or bluffed, i.e. in case apprentices reacted to a bona fide alarm-call vocalized by a tutor in the absence of any predator, or in the presence of a predator belonging to a mismatched class. Putative bluffers would then fall into two separate categories: in the presence of a predator the bluffer acts as a 
sociopath; in the absence of a predator, the bluffer behaves as a prankster. Given that such behaviors have not yet been reported, the original Seyfarth experiments do not show whether vervet monkeys know that they have symbols.

Vocal learning in mammals and birds is dependent on a set of highly specialized brain structures that seem to have evolved under strong common constraints (Gannon, Holloway et al. 1998; Naidich, Hof et al. 2001; Jarvis 2004). In primates, increased cortical compartmentalization correlates with the enhanced sophistication of perceptual, associative and motor functions (Kaas 2004). We propose that the most important brain change that coevolved with vocal language in the Homo lineage is the addition of cortical domains dedicated to the representation of vocal signs and characterized by higher connectivity order, i.e. more and more removed from both the sensory input and the motor output. Such a neural architecture should allow for the combinatorial appearance of meta-symbols (Feldman 2000), i.e. symbols made of parts of many different representations that were likely crucial for the transition between proto-grammars and fully syntactic language.

Acknowledgments: S.R. was partially funded by a Pew Latin American Fellowship, J.Q. was funded by a grant from FAPESP (\#02/09763-2).

\section{References}

Arensburg, B., Tillier, A., 1991. Speech and the Neanderthals. Endeavour 15(1), 26-28.

Arensburg, B., Tillier, A.M., et al., 1989. A Middle Palaeolithic human hyoid bone. Nature 338(6218), 758-760.

Bergman, T.J., Beehner, J.C., et al., 2003. Hierarchical classification by rank and kinship in baboons. Science 302(5648), 1234-1236.

Bickerton, D., 2003. Symbol and structure: a comprehensive framework for language evolution. In: Christiansen, M.H., Kirby, S., (Eds.), Language Evolution: The States of the Art, Oxford University Press.

Cheney, D.L., R., S., 1990. How Monkeys See the World. Chicago, University of Chicago Press.

Cheney, D.L., Seyfarth, R.M., 1998. Why monkeys don't have language. In: Petersen, G., (Ed.) The Tanner Lectures on Human Values.University of Utah Press, Salt Lake City. 19, pp.173-210.

Deacon, T.W., 1997. The symbolic species: The co-evolution of language and brain. New York, W.W. Norton Company. Deacon, T.W., 2003. Universal grammar and semiotic constraints. In: Christiansen, M.H., Kirby, S., (Eds.), Language Evolution.Oxford University Press, Oxford.

Feldman, J., 2000. Minimization of Boolean complexity in human concept learning. Nature 407(6804), 630-633.

Gannon, P.J., Holloway, R.L., et al., 1998. Asymmetry of chimpanzee planum temporale: humanlike pattern of Wernicke's brain language area homolog. Science 279(5348), 220-2.

Griffin, D.R., 1992. Animal Minds. Chicago, The University of Chicago Press.

Hauser, M.D., 1997. The Evolution of Communication. Cambridge, MIT Press.

Hauser, M.D., 2000. A Primate Dictionary? Decoding the Function and Meaning of Another Species' Vocalizations. Cognitive Science 24(3.), 445-475. 
Hebb, D.O., 1949. The Organization of Behavior: A Neuropsychological Theory. New York, John Wiley \& Sons.

Herman, L.M., Kuczaj, S.A.n., et al., 1993. Responses to anomalous gestural sequences by a language-trained dolphin: evidence for processing of semantic relations and syntactic information. J. Exp. Psychol. Gen. 122(2), 184-194.

Jarvis, E.D., 2004. Learned birdsong and the neurobiology of human language. Ann. N. Y. Acad. Sci. 1016, 749-777.

Kaas, J.H., 2004. Evolution of somatosensory and motor cortex in primates. Anat. Rec. A. Discov. Mol. Cell. Evol. Biol. 281(1), 1148-1156.

Kaminski, J., Call, J., et al., 2004. Word learning in a domestic dog: evidence for "fast mapping". Science 304(5677), 16051606.

Langs, R., Badalamenti, A.F., et al., 1996. Two mathematically defined expressive language structures in humans and chimpanzees. Behav. Sci. 41(2), 124-135.

Lieberman, P., 1984. The biology and evolution of language. Cambridge, Harvard University Press.

Lieberman, P., 1998. Eve Spoke: Human language and human evolution. New York, W.W. Norton.

Lieberman, P., Crelin, E.S., 1971. On the speech of Neanderthal man. Linguistic Inquiry 2, 203-222.

Loula, A., Gudwin, R., et al., 2004. Symbolic Communication in Artificial Creatures: an experiment in Artificial Life. In: Bazzan, A., Labidi, S., (Eds.), 17th Brazilian Symposium on Artificial Intelligence - SBIA, São Luis, Brazil. Springer. Lecture Notes in Computer Science 3171, pp.336-345.

Loula, A., Gudwin, R., et al., 2003. A proposal for a synthetic approach of semiotic artificial creatures. In: de Castro, L.N., von Zuben, F.J., (Eds.), Recent Developments in Biologically Inspired Computing.Idea Group Publishing, Hershey, pp.270-300.

Naidich, T.P., Hof, P.R., et al., 2001. Anatomic substrates of language: emphasizing speech. Neuroimaging. Clin. N. Am. 11(2), 305-341.

Noble, W., Davidson, I., 1996. Human evolution, language and mind: a psychological and archaeological enquiry. Cambridge, Cambridge University Press.

Peirce, C.S., 1958. Collected Papers of Charles Sanders Peirce. Cambridge, Mass., Harvard University Press.

Peirce, C.S., 1998. The Essential Peirce: selected philosophical writings. Bloomington, Indiana University Press.

Pepperberg, I.M., 2002. In search of king Solomon's ring: cognitive and communicative studies of Grey parrots (Psittacus erithacus). Brain Behav. Evol. 59(1-2), 54-67.

Queiroz, J., Ribeiro, S., 2002. The biological substrate of icons, indexes and symbols in animal communication. In: Shapiro, M., (Ed.) The Peirce Seminar Papers.Berghahn Books, Oxford, UK. 5, pp.69-78.

Ransdell, J., 1977. Some leading ideas of Peirce's semiotic. Semiotica 19(3), 157-178.

Richards, D.G., Wolz, J.P., et al., 1984. Vocal mimicry of computer-generated sounds and vocal labeling of objects by a bottlenosed dolphin, Tursiops truncatus. J. Comp. Psychol. 98(1), 10-28.

Savage-Rumbaugh, E.S., Rumbaugh, D.M., et al., 1978. Symbolic communication between two chimpanzees (Pan troglodytes). Science 201(4356), 641-4.

Savage-Rumbaugh, S., Romsky, M.A., et al., 1989. Symbol acquisitionand use by Pan troglodytes, Pan paniscus, Homo sapiens. In: Heltne, P.G., Marquardt, L.A., (Eds.), Understanding chimpanzees.Harvard University Press, pp.266295.

Seyfarth, R.M., Cheney, D.L., 1980. The ontogeny of vervet monkey alarm-calling behavior: A preliminary report. Zeitschrift fur Tierpsychologie 54, 37-56.

Seyfarth, R.M., Cheney, D.L., 1986. Vocal development in vervet monkeys. Animal Behaviour 34, 1640-1658.

Seyfarth, R.M., Cheney, D.L., 1997. Some general features of vocal development. in nonhuman primates. In: Social influences on vocal development. In: Snowdon, C.T., Hausberger, M., (Eds.).Cambridge University Press, Cambridge, pp.249-273.

Seyfarth, R.M., Cheney, D.L., et al., 1980. Monkey responses to three different alarm calls: evidence of predator classification and semantic communication. Science 210(4471), 801-3.

Struhsaker, T.T., 1967. Behavior of vervet monkeys and other cercopithecines. New data show structural uniformities in the gestures of semiarboreal and terrestrial cercopithecines. Science 156(779), 1197-203. 
Struhsaker, T.T., Hunkeler, P., 1971. Evidence of tool-using by chimpanzees in the Ivory Coast. Folia Primatol 15(3), 212219.

Vrba, E.S., 1988a. Ecological and adaptive changes associated with early hominid evolution. In: Delson, E., (Ed.) Ancestors: the hard evidence.Alan Liss, New York. 63-71.

Vrba, E.S., 1988b. Late pleistocence climatic events and hominid evolution. In: Grine, F.E., (Ed.) Evolutionary history of the 'robust' australopithecines.Adine de Gruyter, NY. 405-426.

Whiten, A., Goodall, J., et al., 1999. Cultures in chimpanzees. Nature 399(6737), 682-5.

Xia, L., Emmerton, J., et al., 2001. Pigeons (Columba livia) learn to link numerosities with symbols. J. Comp. Psychol. 115(1), 83-91. 